\title{
Expanding anaerobic alkane metabolism in the domain of Archaea
}

\author{
Yinzhao Wang', Gunter Wegener ${ }^{2,3}{ }^{2,3}$ Jialin Hou', Fengping Wang ${ }^{1 *}$ and Xiang Xiao ${ }^{1,4 \star}$
}

\begin{abstract}
Methanogenesis and anaerobic methane oxidation through methyl-coenzyme $M$ reductase (MCR) as a key enzyme have been suggested to be basal pathways of archaea'. How widespread MCR-based alkane metabolism is among archaea, where it occurs and how it evolved remain elusive. Here, we performed a global survey of MCR-encoding genomes based on metagenomic data from various environments. Eleven high-quality mcr-containing metagenomic-assembled genomes were obtained belonging to the Archaeoglobi in the Euryarchaeota, Hadesarchaeota and different TACK superphylum archaea, including the Nezhaarchaeota, Korarchaeota and Verstraetearchaeota. Archaeoglobi WYZ-LMO1 and WYZ-LMO3 and Korarchaeota WYZ-LMO9 encode both the (reverse) methanogenesis and the dissimilatory sulfate reduction pathway, suggesting that they have the genomic potential to couple both pathways in individual organisms. The Hadesarchaeota WYZ-LMO4-6 and Archaeoglobi JdFR42 encode highly divergent MCRs, enzymes that may enable them to thrive on non-methane alkanes. The occurrence of $\mathrm{mcr}$ genes in different archaeal phyla indicates that MCR-based alkane metabolism is common in the domain of Archaea.
\end{abstract}

Since the emergence of life on our planet, anaerobic methane metabolism, including methanogenesis and methane oxidation, has been a crucial element in the Earth's carbon cycle ${ }^{1}$, and both processes are key to the global methane budget. Methanogenic archaea produce $\sim 500-600 \mathrm{Tg}$ of methane per year ${ }^{2}$, whereas anaerobic methane-oxidizing archaea (ANME) oxidize a large portion of methane within the seafloor before it reaches the water column ${ }^{3,4}$. The metabolic pathways of methane formation and anaerobic oxidation of methane are largely identical, as both contain exclusively $\mathrm{C}_{1}$-compound-transforming enzymes that were described originally in the methanogenesis pathway ${ }^{5-7}$.

Among these enzymes, methyl-coenzyme M (CoM) reductase (MCR) plays the crucial role ${ }^{8}$. In methanogens, $\mathrm{MCR}$ reduces $\mathrm{CH}_{3}$ CoM to $\mathrm{CH}_{4}$ (refs. ${ }^{9,10}$ ), whereas in ANMEs, this enzyme activates $\mathrm{CH}_{4}$ to form $\mathrm{CH}_{3}-\mathrm{CoM}$ as the primary intermediate ${ }^{5}$. This canonical MCR type is highly conserved, and the gene encoding the $\alpha$-subunit ( $m c r \mathrm{~A})$ of the enzyme complex has been used as a diagnostic marker for the detection and phylogenetic classification of methanogens and ANMEs ${ }^{11}$. The presence of MCR was thought to be limited to the phylum Euryarchaeota, yet the occurrence of $\mathrm{mcr}$ genes in metagenome-assembled genomes (MAGs) of the Bathyarchaeota ${ }^{12}$ and Verstraetearchaeota ${ }^{13}$ has indicated a much wider distribution of this gene within archaea. In addition, highly divergent MCR types have been identified in two strains of the thermophilic $\mathrm{Ca}$. Syntrophoarchaeum spp. that use the encoded enzymes to activate short-chain alkanes, such as propane and $n$-butane, therewith documenting a first case of a physiological activation of non-methane alkanes using MCR and the production of the corresponding alkylCoM as a primary intermediate ${ }^{14}$. None of the known MCR-based alkane-oxidizing archaea growing under sulfate-reducing conditions possess genes encoding enzymes for dissimilatory sulfate reduction; hence, all of them rely on syntrophic interactions with sulfate-reducing partner bacteria ${ }^{14-17}$. By contrast, some ANME-2d contain nitrite or nitrate reductases, or may transfer electrons to metal oxides, and hence may not require partner bacteria when growing under these conditions ${ }^{18,19}$.

In this study, we aimed to provide a larger overview of the distribution of MCR-based alkane metabolism in archaea from different environments. Thus, we established a database of known representative McrA protein sequences as a reference $(n=153$; Supplementary Table 1), and with this list as a basis, the Joint Genome Institute (JGI) metagenomic protein databases (January 2017) were used as queries for screening homologous sequences (identity $>30 \%, e<10^{-20}$, coverage $>75 \%$ ). Sixty-four metagenomic data sets produced on Illumina HiSeq 2000 or 2500 platforms containing McrA sequences were further analysed (Supplementary Table 2). These data sets were then downloaded from the NCBI Sequence Read Archive public database, reassembled, binned and annotated as described in the Methods section. A phylogenetic analysis of the McrA subunits retrieved from these metagenomes shows that they cluster with those of the Euryarchaeota phylum, including ANME-1, ANME2a/b, ANME-2c, ANME-2d, ANME-3, Ca. Syntrophoarchaeum spp. and most of the known methanogens, and with those of non-Euryarchaeota phyla, including the Verstraetearchaeota and Bathyarchaeota. The majority of the McrA sequences that affiliate with ANME homologues show high identities to the known McrA sequences, whereas nearly half of the McrA sequences affiliating with the Verstraetearchaeota and $\mathrm{Ca}$. Syntrophoarchaeum spp./ Bathyarchaeota have less than $80 \%$ and $60 \%$ identities to the known sequences, respectively (Supplementary Table 3 ). This suggests that many of these sequences come from unknown microorganisms.

From the metagenomic sequences of 3 geothermal environments, we recovered 11 high-quality $m c r$-containing MAGs that contained divergent $m c r$ genes (Table 1 and Supplementary Table 2). All MAGs were checked for lineage-specific (149-228) and Archaea (149) marker proteins to evaluate their completeness and contamination (Supplementary Table 4). The completeness of all reported MAGs was higher than $80 \%$, whereas their contamination was below $2 \%$. The GC contents, tetranucleotide frequencies and codon usage patterns of the mor gene containing contigs show high congruence with their respective MAGs (Supplementary Figs. 1-3). This strongly

'State Key Laboratory of Microbial Metabolism, School of Life Sciences and Biotechnology, Shanghai Jiao Tong University, Shanghai, China. ${ }^{2}$ Max Planck Institute for Marine Microbiology, Bremen, Germany. ${ }^{3}$ MARUM, Center for Marine Environmental Sciences, University of Bremen, Bremen, Germany. ${ }^{4}$ State Key Laboratory of Ocean Engineering, Ocean and Civil Engineering, Shanghai Jiao Tong University, Shanghai, China. *e-mail: fengpingw@sjtu.edu.cn; zjxiao2018@sjtu.edu.cn 
Table 1 | MAGs described in this study and their main potential metabolic features

\begin{tabular}{llll} 
MAG name & Sampling location & MCR group & Potential alkane metabolic pathways \\
\hline Archaeoglobi WYZ-LMO1 & Washburn Spring, WY, USA & II & MAO coupled to DSR in one cell or methanogenesis \\
Archaeoglobi WYZ-LMO2 & Obsidian Pool, WY, USA & II & Methanogenesis from $\mathrm{CO}_{2}$ and $\mathrm{H}_{2}$ or $\mathrm{MAO}_{\text {coupled to DSR }}$ \\
Archaeoglobi WYZ-LMO3 & Obsidian Pool, WY, USA & II & MAO coupled to DSR in one cell or methanogenesis \\
Archaeoglobi JdFR-42 & Juan de Fuca Ridge, Pacific & III & MAO coupled to nitrate reduction or external $\mathrm{e}^{-}$sinks \\
Hadesarchaeota WYZ-LMO4 & Jinze Hot Spring, Yunnan, China & III & MAO with unknown or external $\mathrm{e}^{-}$sink or alkane production \\
Hadesarchaeota WYZ-LMO5 & Jinze Hot Spring, Yunnan, China & III & MAO with unknown or external $\mathrm{e}^{-}$sink or alkane production \\
Hadesarchaeota WYZ-LMO6 & Washburn Spring, WY, USA & III & MAO with unknown or external $\mathrm{e}^{-}$sink or alkane production \\
Nezhaarchaeota WYZ-LMO7 & Jinze Hot Spring, Yunnan, China & II & Methanogenesis from $\mathrm{CO}_{2}$ and $\mathrm{H}_{2}$ \\
Nezhaarchaeota WYZ-LMO8 & Jinze Hot Spring, Yunnan, China & II & Methanogenesis from $\mathrm{CO}_{2}$ and $\mathrm{H}_{2}$ \\
Korarchaeota WYZ-LMO9 & Washburn Spring, WY, USA & II & MAO coupled to sulfite reduction; methanogenesis from \\
Verstraetearchaeota WYZ-LMO10 & Washburn Spring, WY, USA & II & methyl groups and $\mathrm{H}_{2}$ or reduced sulfur species as an $\mathrm{e}^{-}$source \\
Verstraetearchaeota WYZ-LMO11 & Washburn Spring, WY, USA & II & Methanogenesis from methyl groups and $\mathrm{H}_{2}$ \\
\hline DSR, mesis from methyl groups and $\mathrm{H}_{2}$
\end{tabular}

DSR, dissimilatory sulfate/sulfite reduction; MAO, MCR-based alkane oxidation. aOrigin of this bin from ref. ${ }^{5}$.

indicates that these contigs were correctly assigned into MAGs. By contrast, these measures show clear differences when comparing them with genomes of other closely related archaea. The 11 MAGs with divergent $\mathrm{mcr}$ genes and reference archaea genomes from the NCBI genome database were used to construct phylogenetic trees based on a concatenated set of 37 marker genes (Supplementary Table 5) and 16S rRNA gene (Fig. 1a,b). According to these analyses, the MAGs belong to the Euryarchaeota, Hadesarchaeota ${ }^{20}$, Korarchaeota ${ }^{21}$, Verstraetearchaeota ${ }^{13}$ and an additional clade in the TACK superphylum (Table 1).

Three MAGs (WYZ-LMO1-3) clustered within the class Archaeoglobi, neighbouring with Archaeoglobus fulgidus (Fig. 1a,b). They showed whole genome average amino acid identities (AAIs) of $65 \%$ and $16 \mathrm{~S}$ rRNA gene identities of $\sim 85-94 \%$ to their most closely related cultured Archaeoglobaceae strains (Supplementary Tables 6 and 7). Three MAGs (WYZ-LMO4-6) clustered within the Hadesarchaea and neighboured with MSBL-1 archaea (Fig. 1a,b). Whereas Hadesarchaea have been previously suggested to cluster within the Euryarchaeota ${ }^{20}$, recent analyses suggested the classification of Hadesarchaea as a separate phylum Hadesarchaeota, probably branching at the base of the Asgard/TACK superphyla ${ }^{22,23}$. Our results suggest that the Hadesarchaeota branch with Euryarchaeota. Yet, the bootstrap support for this is low, hence further analyses with additional archaeal lineages, including a congruent placement of the root of archaea ${ }^{24-26}$, may be required to finally resolve the phylogenetic placement of the Hadesarchaeota. Two MAGs (WYZ-LMO7 and WYZ-LMO8) form a deep-branching cluster within the TACK superphylum. Although the partial 16S rRNA gene sequences ( 900 bp) from MAGs WYZ-LMO7 and WYZ-LMO8 shared $\sim 89 \%$ identities with the Thermoprotei strains in Crenarchaeota (Fig. 1b), the AAIs with the closest reference genomes of the Verstraetearchaeota, Geothermarchaeota and Crenarchaeota were only $\sim 48-50 \%$. Moreover, both genomic protein sequences and MCR coding sequences have very low average identities (on average $<50 \%)$ to the known protein sequences in the NCBI nr database (Supplementary Fig. 4), indicating that these MAGs may represent an additional lineage of alkane-metabolizing archaea. Although, more genomes from representatives of this lineage are required to determine its taxonomic level, here, we temporarily named it Nezhaarchaeota, after Nezha, an immortal in Chinese mythology. One MAG (WYZ-LMO9) clustered closely with the Korarchaeota (Fig. 1a,b) but shared only 58\% AAI and $~ 92 \% 16 \mathrm{~S}$ rRNA gene identity with the $\mathrm{Ca}$. Korarchaeum cryptofilum OPF8, suggesting that MAG WYZ-LMO9 might represent an additional family in the phylum Korarchaeota. Two MAGs (WYZ-LMO10 and WYZLMO11) are affiliated with the Verstraetearchaeota, but showed $<65 \%$ AAI with all published Verstraetearchaeota genomes and also displayed large distances in the phylogenomic tree. The MAGs WYZ-LMO10 and WYZ-LMO11 also share low AAI ( 65\%) between each other and show large differences in their average genomic GC content (WYZ-LMO10: 50.3\%; WYZ-LMO11: 28.5\%).

Our study extends the known distribution of $\mathrm{mcr}$ genes in the Archaea domain and identifies additional lineages of potential alkane-metabolizing archaea within the Archaeoglobi in the Euryarchaeota, Hadesarchaeota and TACK superphylum. The highly similar tree topologies for all three subunits clearly indicate a coevolution of the encoded protein subunits (Fig. 2). Based on phylogenetic analyses, the MCR sequences were categorized into three groups (I, II and III). Group I contains sequences of the canonical MCRs including those originating from the Euryarchaeota (Fig. 2). Group II is between the canonical and divergent MCRs and is dominated by sequences assigned to archaea of the TACK superphylum, yet it also includes the MCRs of Archaeoglobi WYZ-LMO1-3 (Fig. 2). Group III represents the putative multi-carbon alkane metabolizing MCRs, and includes not only divergent MCR sequences of $C a$. Syntrophoarchaeum spp. ${ }^{14}$ and the Bathyarchaeota ${ }^{12}$ but also those of the Hadesarchaeota and one Archaeoglobi MAG (Archaeoglobi JdFR-42, NCBI genomic database; Fig. 2).

The Archaeoglobi MAGs WYZ-LMO1-3 contain $\mathrm{mcr}$ genes of group II MCR and complete methanogenesis pathways (Fig. 3a,b and Supplementary Tables 8 and 9), whereas two MAGs (WYZLMO1 and WYZ-LMO3) additionally possess genes encoding the complete sulfate reduction pathway, including genes for sulfate adenylyltransferase, adenylylsulfate reductase (AprAB) and dissimilatory sulfite reductase (DsrAB). We also identified genes encoding the DsrC protein and the DsrMKJOP complex, which are regularly found in sulfate reducers. The DsrA sequences from WYZ-LMO1 and WYZ-LMO3 are closely related to those of the reducingtype enzymes in the Firmicutes (Supplementary Fig. 5). Many Archaeoglobi probably acquired the $d s r \mathrm{AB}$ and aprAB genes from a bacterial donor early during the evolution of this lineage and thus obtained the ability to perform dissimilatory sulfate reduction ${ }^{27,28}$. For decades, it was believed that sulfate-dependent methane oxidation requires the interaction of anaerobic methane-oxidizing archaea and sulfate-reducing partner bacteria ${ }^{29}$. However, based on the genomic potential described here, some Archaeoglobi might be 


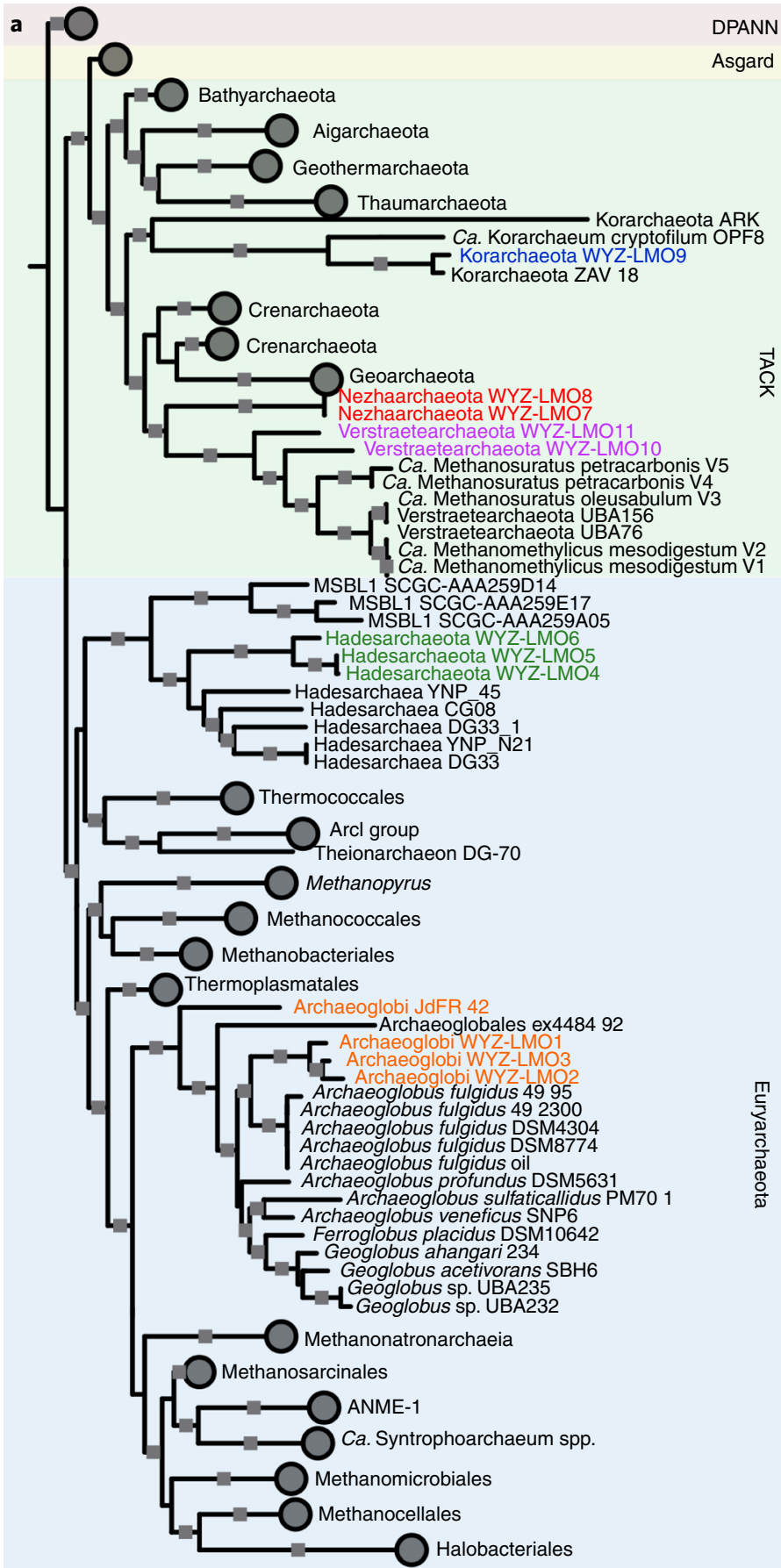

b

$\int^{0}-E_{-5}^{-}$

MSBL1 SCGC-AAA259D14 MSBL1 SCGC-AAA259A05 MSBL1 SCGC-AAA259E17 Hadesarchaea YNPNA N21

Hadesarchaeota WYYZ-LMO6 Hadesarchaeota WYZ-LMO5 T Korarchaeota SRI-306

Ca. Korarchaeum cryptofilum OPF8

Asgard

(1)

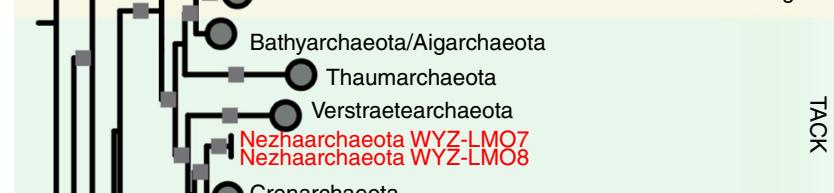

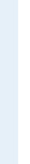

Fig. 1 | Classification of the 12 described MAGs. a, Phylogenomic affiliation of the MAGs based on 37 conserved protein sequences and using 233 representative archaeal genomes from 4 superphyla. b. Phylogenetic affiliation of the MAGs and representative archaea based on their $165 \mathrm{SRNA}$ genes. Both alignments were based on MAFFT and then filtered with trimAl, and the trees were built by the IQ-Tree method with the model $L G+C 60+F+G$ or $\mathrm{HKY}+\mathrm{F}+\mathrm{G}$ with 1,000 bootstrap replicates. The phylogenetic trees were rooted at the DPANN superphylum and all four superphyla were assigned different background colours. Distinct mcr genes containing archaea are shown with coloured text (the Archaeoglobi WYZ-LMO1-3/JdFR-42, orange; Hadesarchaeota WYZ4-6, green; Nezhaarchaeota WYZ-LMO7 and WYZ-LMO8, red Korarchaeota WYZ-LMO9, blue; Verstraetearchaeota WYZ-LMO10 and WYZ-LMO11, purple).

able to perform sulfate-dependent methane oxidation in single cells. Yet, we cannot rule out the possibility that these Archaeoglobi are also methanogens, although no complete hydrogenase was found in their genomes. The Archaeoglobi MAG WYZ-LMO2 does not have the $d s r \mathrm{AB}$ and aprAB genes. Hence, as alkane oxidizer, it would require an electron-accepting partner organism. However, the Archaeoglobi MAG WYZ-LMO2 contains a complete Ni-Fe hydrogenase gene set that resembles $\mathrm{F}_{420}$-non-reducing hydrogenase (MvhADG). This enzyme is usually found in methanogens and can produce reduced ferredoxin and reduced coenzyme M (CoMSH) by consuming hydrogen ${ }^{30}$. Hence, the Archaeoglobi WYZ-LMO2 might be instead a hydrogenotrophic methanogen. Only cultivation of representative organisms will be able to verify the genome-based hypotheses on these Archaeoglobi. 


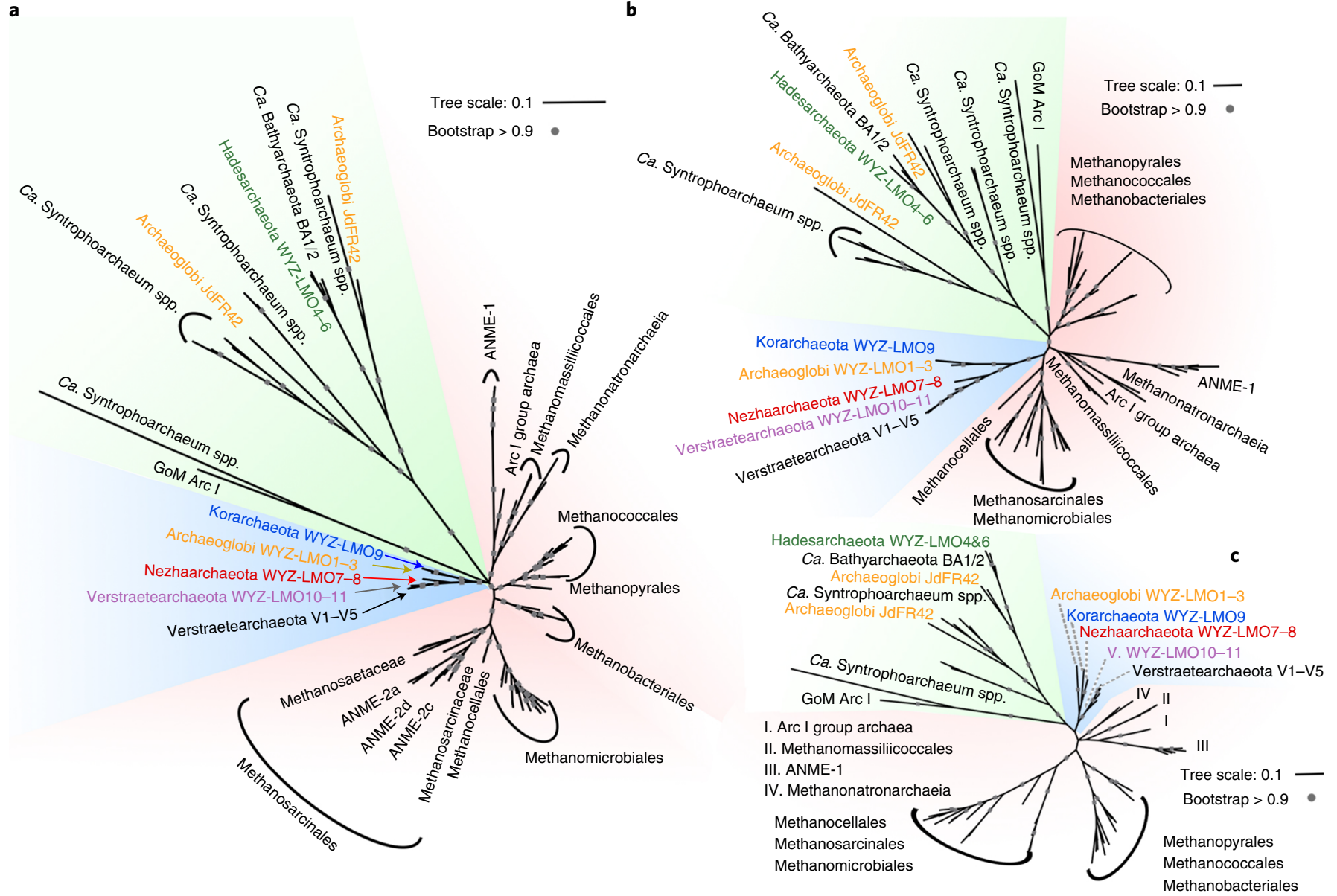

Fig. 2 | Phylogenetic affiliations of the McrA, McrB and McrG protein sequences of the 12 studied archaeal MAGs. a-c, The phylogenetic tree was constructed based on the alignments of McrA with 461 aligned positions (a), McrB with 351 aligned positions (b) and McrG with 221 aligned positions (c). Alignments were generated using MAFFT and then filtered with trimAl, and the trees were built by the IQ-Tree method with the model $L G+C 60+F+G$ with 1,000 bootstrap replicates. The tree branches were classified into three distinct MCR groups: canonical euryarchaeotal MCRs (group I, red shaded background), the MCRs of group II dominated by sequences of the TACK superphylum archaea but including the Archaeoglobi WYZ-LMO1-3 Mcr sequences (group II, blue shaded background) and the putative multi-carbon alkane oxidization MCRs (group III, green shaded background) that seem to be laterally transferred between different Archaeoglobi, Hadesarchaeota, Bathyarchaeota and Ca. Syntrophoarchaeum spp. Previously unidentified MCR protein sequences are also coloured (the Archaeoglobi WYZ-LMO1-3/JdFR-42, orange; Hadesarchaeota WYZ4-6, green; Nezhaarchaeota WYZ-LMO7 and WYZ-LMO8, red; Korarchaeota WYZ-LMO9, blue; Verstraetearchaeota WYZ-LMO10 and WYZ-LMO11, purple).

Strikingly, we found an Archaeoglobi MAG that contains two highly divergent group III morgene sets in the NCBI genome database (Archaeoglobi JdFR-42; Fig. 3c and Supplementary Table 8). Both $m c r$ gene sets are similar to those found in $\mathrm{Ca}$. Syntrophoarchaeum spp. In Ca. Syntrophoarchaeum spp., the encoded MCR complexes have been shown to activate the multi-carbon alkanes propane and $n$-butane ${ }^{14}$. Moreover, MAG JdFR-42 encodes a Wood-Ljungdahl pathway and a pathway for fatty acid oxidation, including acyl-CoA dehydrogenase (FadE), crotonase (FadB1), 3-hydroxyacyl-CoA dehydrogenase (FadB2) and acetyl-CoA acetyltransferase (AtoB). These pathways are similar to those found in $\mathrm{Ca}$. Syntrophoarchaeum spp. Hence, we suggest that Archaeoglobi JdFR-42 is another multicarbon alkane degrader. Similar to $\mathrm{Ca}$. Syntrophoarchaeum spp., MAG JdFR-42 does not carry genes encoding enzymes for sulfate reduction, such as $d s r \mathrm{AB}$ and $a p r \mathrm{AB}$. By contrast, its genome contains genes encoding nitrate reductase/nitrite oxidoreductase (NarGHI), periplasmic nitrate reductase (NapA), nitrate/nitrite transporter $(\mathrm{NrtP})$ and the nitrogenase iron protein $\mathrm{NifH}$ similar to ANME-2d, thus might use nitrate/nitrite as electron sinks. Alternatively, Archaeoglobi JdFR-42 may have electron-accepting partner bacteria as shown for $\mathrm{Ca}$. Syntrophoarchaeum spp.
The Hadesarchaeota MAGs WYZ-LMO4, WYZ-LMO5 and WYZ-LMO6 (Fig. 3d and Supplementary Tables 8 and 9) contain group III $\mathrm{mcr}$ genes that cluster with those of the $\mathrm{Ca}$. Syntrophoarchaeum spp./Bathyarchaeota group. All three MAGs have $\beta$-oxidation pathways and they all contain the WoodLjungdahl pathway, while lacking genes encoding sulfate or nitrate reduction. The published MAGs of Hadesarchaea (YNP_N21, YNP_45, DG-33 and DG-33-1) do not contain $m c r$ genes and thus have been suggested to thrive as carbon monoxide and hydrogen oxidizers that can fix $\mathrm{CO}_{2}$ in subsurface environments ${ }^{20}$. However, the gene repertoire of the Hadesarchaeota obtained in this study suggests that they may oxidize short-chain alkanes similar to $\mathrm{Ca}$. Syntrophoarchaeum spp. ${ }^{14}$.

The Nezhaarchaeota MAGs WYZ-LMO7 and WYZ-LMO8 (Fig. 3e and Supplementary Tables 8 and 9) contain $\mathrm{mcr}$ genes of group II MCR. These archaea also encode the complete methanogenesis pathway and two hydrogenases, coenzyme $\mathrm{F}_{420}$ hydrogenase (FrhABDG) and ferredoxin-dependent hydrogenase (ECH), which are considered to act in coenzyme $\mathrm{F}_{420}$ and ferredoxin recycling, respectively. The MAGs WYZ-LMO7 and WYZ-LMO8 contain formate dehydrogenase-like genes next to a heterodisulfide reductase 
a

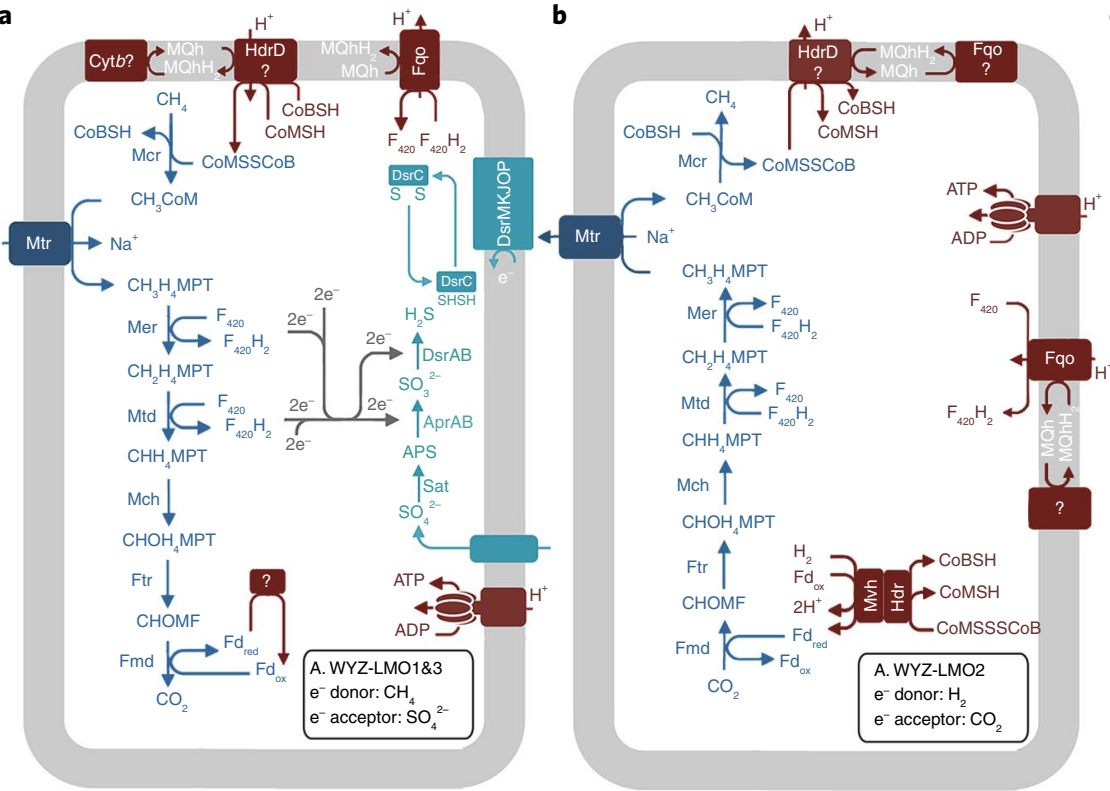

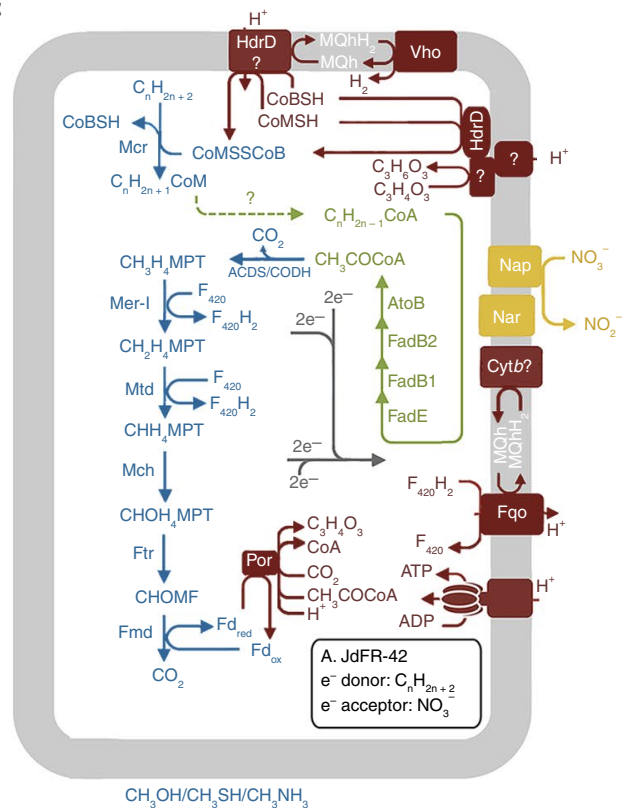

$\mathbf{f}$

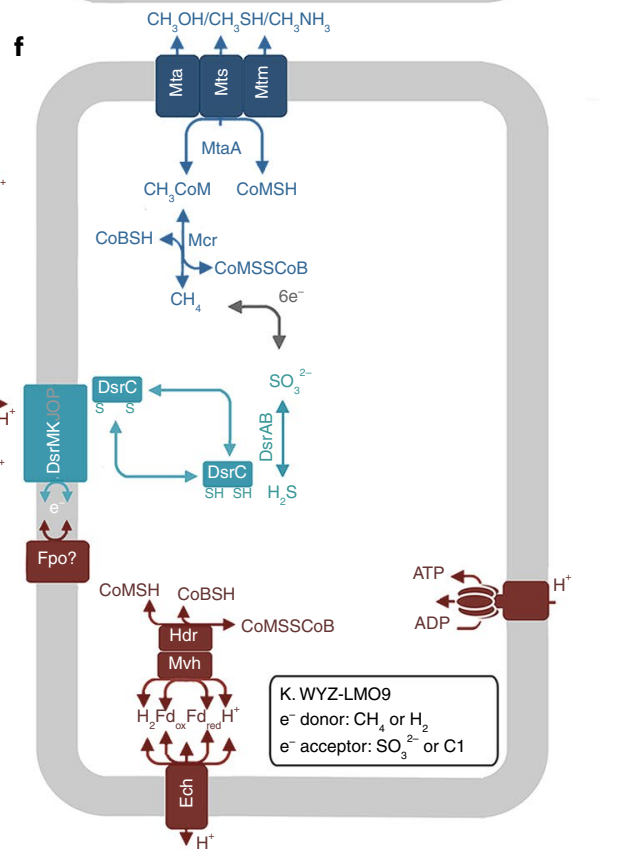

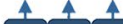
CoM COMSH Mcr d

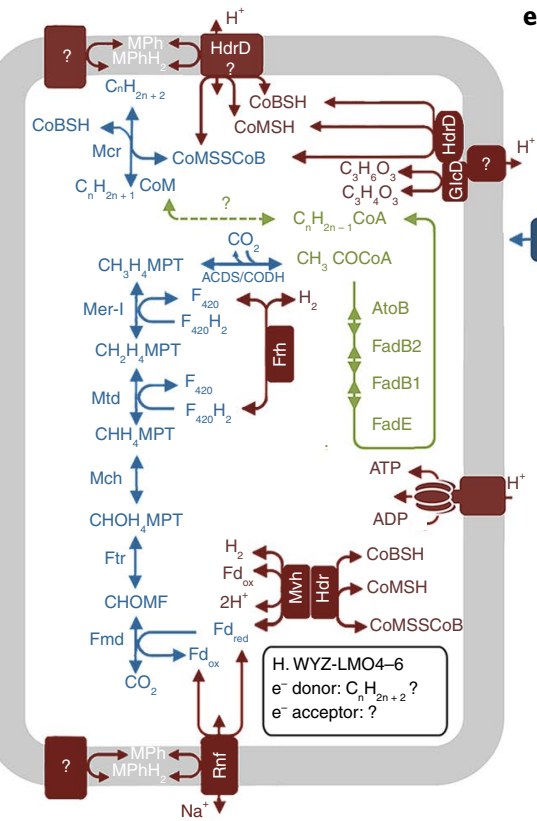

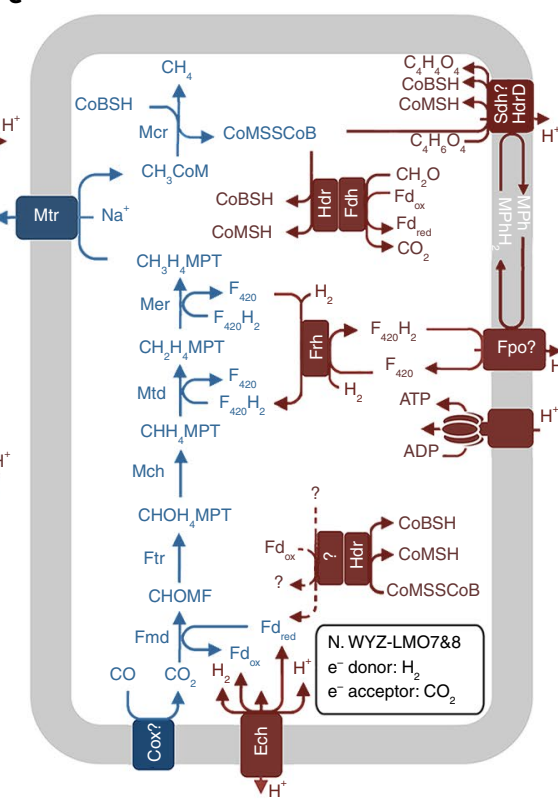

Fig. 3 | Alkane metabolic schemes of the studied MAGs with previously unidentified $\mathbf{m c r}$ genes. a-c, The MAGs demonstrate the large metabolic diversity of mcr-containing Archaeoglobi. WYZ-LMO1 (88.89\% genome completeness) and WYZ-LMO3 (87.40\% completeness) are probably methane oxidizers capable of sulfate reduction (a). WYZ-LMO2 ( $97.60 \%$ completeness), with a hydrogenase mvh gene cluster but no sulfate-reducing genes, is probably a methanogen or partner-bacterium-dependent methane oxidizer (b). JdFR-42 (99.84\% completeness) contains the multi-carbon alkane oxidation group III mcr gene cluster, genes for fatty acid degradation and genes for nitrate/nitrite reduction; hence, it is probably a nitrate/nitrite- or partner-bacterium-dependent alkane oxidizer (c).d, The MAGs of Hadesarchaeota WYZ-LMO4-6 (88.89\%, 83.26\% and 92.37\% completeness, respectively) encode sets of enzymes for multi-carbon alkane metabolism; the absence of reductive pathways suggests a need for partner bacteria. e, The Nezhaarchaeota WYZ-LMO7 (93.90\% completeness) and WYZ-LMO8 (97.20\% completeness) contain the genes for hydrogenotrophic methanogenesis. f, The Korarchaeota WYZ-LMO9 has both $\mathrm{mcr}$ and dsr genes but lacks the Wood-Ljungdahl pathway, it might also oxidize methane with sulfite reduction; alternatively, it is probably a methylotrophic methanogen that uses electrons from sulfide or hydrogen oxidation. Question marks indicate unknown enzymes and substrates; dashed arrows indicate unknown reactions. Dark blue reactions are those involved in hydrocarbon metabolism; light blue reactions are those involved in dissimilatory sulfate reduction; red reactions are those involved in energy conservation; green reactions are those involved in $\beta$-oxidation; and yellow reactions are those involved in dissimilatory nitrate reduction. ACDS, acetyl-CoA decarbonylase/synthase; $\mathrm{CODH}$, carbonmonoxide dehydrogenase; Cox, carbon monoxide dehydrogenase; Cytb, cytochrome b reductase; CHOMF, formylmethanofuran; Fmd, formylmethanofuran dehydrogenase; $\mathrm{Fpo}, \mathrm{F}_{420} \mathrm{H}_{2}$ dehydrogenase; $\mathrm{Fqo}, \mathrm{F}_{420} \mathrm{H}_{2}$ :quinone oxidoreductase; Ftr, formylmethanofuran-tetrahydromethanopterin $\mathrm{N}$-formyltransferase; GIcD, glycolate oxidase; Mch, methenyltetrahydromethanopterin cyclohydrolase; Mer, 5,10-methylenetetrahydromethanopterin reductase; Mer-I, 5,10-methylenetetrahydromethanopterin reductase-like enzyme; Mtd, methylenetetrahydromethanopterin dehydrogenase; NarDHI, nitrate reductase/ nitrite oxidoreductase; Por, pyruvate ferredoxin oxidoreductase; Rnf, $\mathrm{Na}^{+}$-translocating ferredoxin: $\mathrm{NAD}^{+}$oxidoreductase; Sat, sulfate adenylyltransferase; Vho, methanophenazine-dependent hydrogenase; APS, adenosine phosphosulfate; $\mathrm{H}_{4} \mathrm{MPT}$, tetrahydromethanonopterin; CoMSSCoB, heterodisulfide coenzyme B coenzme M; $\mathrm{Fd}_{\text {ox }}$ oxidized ferrodoxin; $\mathrm{Fd}_{\text {red }}$, reduced type ferrodoxin; MPh, methanophenazine; MQh, menaquinone; MPT, methanopterin. 
(HdrABC) with Fe-S-cluster-containing components. Moreover, two $h d r \mathrm{D}$ genes are located near succinate dehydrogenase-like genes and FAD/FMN-containing dehydrogenase genes; together, these enzymes could be used for the reduction of the heterodisulfide coenzyme B coenzme M (CoBS-SCoM) to reduced coenzyme $\mathrm{M}$ and reduced coenzyme $\mathrm{B}$. Thus, these Nezhaarchaeota are most likely hydrogenotrophic methanogens.

The Korarchaeota MAG WYZ-LMO9 (Fig. 3f and Supplementary Tables 8 and 9) also contains group II $m c r$ genes, as well as the sulfite reductase $(d s r \mathrm{AB})$ genes that are related to those of the Firmicutes (Supplementary Fig. 5). In case the encoded DSR enzyme complex is used in an oxidative direction, electrons liberated in the transformation of sulfide to sulfite could be used for the production of methane from methyl groups. Conversely, a reductive use of the DSR might also be likely, as it would accept the electron from methane consumption. MAG WYZ-LMO9 lacks the genes for the upstream part of the methanogenesis pathway, but encodes methyltransferases, such as (methyl-Co(III) methanol-specific corrinoid protein):CoM methyltransferase (MtaA), methylthiol:CoM methyltransferase (MtsA) and methylamine-corrinoid protein co-methyltransferase (MtmB), suggesting that it may use methylated compounds for methane production or vice versa. MAG WYZ-LMO9 also contains genes encoding the HDR complex for the regeneration of free $\mathrm{CoMSH}$ and $\mathrm{CoBSH}$, and encodes four potential sets of hydrogenases (MvhADG, FrhDG and two putative Ni-Fe hydrogenase: $\mathrm{NiFe}$-group-1g and NiFe-group-4b).

The Verstraetearchaeota MAGs WYZ-LMO10 and WYZLMO11 (Supplementary Tables 8 and 9) also contain $\mathrm{mcr}$ genes of the group II MCR. They miss the upstream part of the methanogenesis pathway, but contain genes encoding MtaA, MtsA and $\mathrm{MtmB}$, indicating that they are likewise methylotrophic methanogens. MAGs WYZ-LMO10 and WYZ-LMO11 also contain genes encoding the HdrDE complex for the regeneration of free CoMSH and $\mathrm{CoBSH}$, as well as the $\mathrm{ECH}$ complex and energy-converting hydrogenase (EHB) for ferredoxin recycling. The combination of these pathways may allow the Verstraetearchaeota to thrive as methylotrophic methanogens, yet they would require an electron donor, presumably molecular hydrogen as suggested before ${ }^{13}$.

The genes encoding MCR-based alkane metabolism have now been identified in the Euryarchaeota, Hadesarchaeota and different clades of the TACK superphylum. Our phylogenetic analysis of all three subunits of the MCR complex showed that the canonical Euryarchaeota Mcr sequences (group I MCR) form at least three distinct monophyletic clusters (euryarchaeotal-cluster I includes the Methanobacteriales, Methanococcales and Methanopyrales; euryarchaeotal-cluster II includes the Methanocellales, Methanomicrobiales and Methanosarcinales; euryarchaeotal-cluster III includes the Methanomassiliicoccales, Arc I group (Methanofastidiosales), Methanonatronarchaeia and ANME-1). The MCR in group II forms a well-supported monophyletic cluster next to group I and contains MCR sequences from the TACK superphylum archaea, yet includes the MCRs of the Archaeoglobi MAGs WYZ-LMO1-3. Besides this exception, the phylogeny of both the group I and group II MCRs are consistent with the topologies from phylogenomic and 16S rRNA gene phylogenetic analyses (Figs. 1 and 2). Two scenarios could explain the presence of the euryarchaeotal Archaeoglobi Mcr sequences in group II MCR. One possibility is that a common ancestor of the Euryarchaeota and TACK archaea already contained a $m c r$ gene cluster that was vertically transferred into the two emerging superphyla, which since then would have evolved independently. In this scenario, archaea from the Archaeoglobi might have obtained the group II $m c r$ genes by horizontal gene transfer from a TACK archaeon. In a second scenario, one ancestor of the TACK archaea might also have acquired its MCR after the divergence of the two superphyla, possibly from the $\mathrm{mcr}$-containing Archaeoglobi lineage.
The group III MCRs are present in different phyla, including the Euryarchaeota, Hadesarchaeota and Bathyarchaeota. The wide phylogenetic distribution and the low number of available MAGs with this MCR type may allow limited predictions on its origin. Nevertheless, the McrA and McrG proteins of the group III MCR also show close affiliations with the group II MCR, as well as with euryarchaeotal-cluster III MCR in group I (Fig. 2a,c). One possible explanation for the distribution of group III $\mathrm{mcr}$ genes in different archaeal phyla is horizontal gene transfer, which allowed several formerly most likely methanogenic or methane-oxidizing archaea to become multi-carbon alkane oxidizers. The high similarities between MCR and genome GC contents, tetranucleotide frequencies and codon usages (Supplementary Figs. 1-3) show that this horizontal gene transfer should have appeared early in the evolution of these organisms. Alternatively, owing to positive natural selection, several methanogens or methane oxidizers might have developed multi-carbon-type MCRs by convergent evolution of canonical MCRs. Genomic information from additional $\mathrm{mcr}$-containing organisms should be required to further evaluate whether the wide distribution of $m c r$ genes in the domain of Archaea is due to mechanisms of horizontal or vertical gene transfer and to identify the most ancestral mcr-containing archaeon.

Our study provides a global-scale metagenome-based analysis of the diversity of $m c r$-containing microorganisms. Particularly from geothermal environments, we retrieved mcr-containing MAGs of representatives affiliated with the Archaeoglobi in the Euryarchaeota, Hadesarchaeota, Korarchaeota, Nezhaarchaeota and MAGs of recently described Verstraetearchaeota that probably perform (reverse) methanogenesis and multi-carbon alkane oxidation, and we provide genomic evidence for the coexistence of methane metabolism and sulfate reduction in single MAGs of Archaeoglobi and Korarchaeota. This suggests a greater importance of archaea in global carbon balancing. However, in vitro cultivation of representatives of the here described organisms and additional genomes of alkane-metabolizing archaea will be required to validate the metabolic hypotheses suggested in this study.

\section{Methods}

Data selection and treatments of raw sequencing reads. Predicted protein sequences from high-throughput metagenomes were downloaded from the JGI database (https://genome.jgi.doe.gov; January 2017). To identify metagenomic data sets containing MCR-based alkane metabolism-related genes, the metagenomic protein sequences were queried against a local McrA protein database $(n=153$ Supplementary Table 1) containing sequences from the known methanogens, ANMEs and multi-carbon alkane-oxidizing archaea (sequences were retrieved from the NCBI database: https://www.ncbi.nlm.nih.gov), using DIAMOND ${ }^{31}$ version 0.8 .28 .90 (identity $>30 \%$, coverage $>75 \%, e<1 \times 10^{-20}$ ). This screening identified $64 \mathrm{mcr}$ A gene-containing data sets (Supplementary Table 2), for which the metagenomic raw sequencing reads data were downloaded from the NCBI Sequence Read Archive public database (https://www.ncbi.nlm.nih.gov/sra/). Raw sequencing reads were trimmed using the Sickle algorithm version 1.33 (https:// github.com/najoshi/sickle), and trimmed reads were assembled using MEGAHIT ${ }^{32}$ version 1.0.6-hotfix1 with $\mathrm{k}$-min 27 and $\mathrm{k}$-max 127. The sequence coverage of each contig was determined by mapping the original trimmed reads to the contigs using Bowtie ${ }^{33}$ version 2.2.8 with parameter --very-sensitive. The assembled data were subjected to open reading frame (ORF) prediction with Prodigal ${ }^{34}$ version 2.6.3 with parameter -meta. The predicted ORFs were searched against the NCBI nr protein database (March 2017) and the web-based KEGG portal GhostKOALA (metagenome annotation) and $\mathrm{KEGG}^{35}$ mapping for primary annotation.

Binning of genomes and evaluations. The assembled metagenomic sequences were binned based on the tetranucleotide frequency dimensionality reduction by $\mathrm{ESOM}^{36}$, based on abundance information using $\mathrm{MaxBin}^{37}$ version 2.2.4 with the run_MaxBin.pl script and based on abundance and tetranucleotide frequency using MetaBAT ${ }^{38}$ version 2.12 .1 with $1 \mathrm{~kb}$ (or $1.5 \mathrm{~kb}$ ) and $3 \mathrm{~kb}$ as contig length cut-offs. The MAGs with the highest completeness and lowest contamination as evaluated using CheckM ${ }^{39}$ version 1.0.7 using lineage-specific marker genes with parameter lineage_wf were considered for further analyses. The selected MAGs were then refined with the mmgenome ${ }^{40}$ package version 0.6 .3 . Contigs that did not match the $95 \%$ confidence interval for depth and GC contents as calculated using the $\mathrm{R}$ function 'quantile' were removed. In addition, the annotation results 
of each contig were checked, and potential contamination (defined as the entire contig was annotated as another microorganism with protein sequence identities higher than $80 \%$ ) was manually removed as suggested by Bowers et al. ${ }^{41}$. The correct assignment of the $m c r$ and $d s r$ containing contigs to a certain MAG was additionally evaluated by comparing the GC contents, tetranucleotide frequencies and codon usages of the $m c r$ - and $d s r$-containing contigs with those of their complete MAGs and genomes of methanogens, as well as closely related archaea. In detail, for selecting the most related methanogen genomes, we compared the McrA protein sequences from each of the MAGs to the NCBI refseq database to determine the highest-scoring McrA hits and downloaded the genome of the respective organisms, namely, the Archaeoglobi WYZ-LMO1-3 with Methanocaldococcus bathoardescens JH146, the Hadesarchaeota WYZLMO4-6 with Bathyarchaeota archaeon BA2, the Nezhaarchaeota WYZ-LMO7 and WYZ-LMO8 with Methanopyrus sp. KOL6, the Korarchaeota WYZ-LMO9 with Methanocaldococcus vulcanius M7 and the Verstraetearchaeota WYZ-LMO10 and WYZ-LMO11 with Methanosuratus petracarbonis V4. For selecting genomes of archaea related to the MAGs in the current study, the genomes that closely clustered to the MAG based on the phylogenomic analysis were considered, that is, the Archaeoglobi WYZ-LMO1-3 with A.fulgidus DSM 4304, the Hadesarchaeota WYZ-LMO4-6 with Hadesarchaea archaeon YNP_45, the Nezhaarchaeota WYZ LMO7 and WYZ-LMO8 with M. petracarbonis V4, the Korarchaeota WYZ-LMO9 with Korarchaeum cryptofilum OPF8, the Verstraetearchaeota WYZ-LMO10 and WYZ-LMO11 with $M$. petracarbonis V4. The average GC contents were calculated using the sum of cytosine and thymine divided by the sum of cytosine, thymine, adenine and guanine of contigs or genomes. Tetranucleotide frequencies were calculated by the Perl script tetramer_freqs_esom.p ${ }^{36}$. The tetranucleotide frequencies of all $m c r$ - and $d s r$-containing contigs, the studied MAGs, their related archaea and their related methanogens were displayed by Principal Component Analysis (PCA) for dimensionality reduction as calculated by R function 'prcomp'. Codon usage frequencies were calculated at http://www.bioinformatics.org/sms2/ codon_usage.html and the codon usage distances were shown as the difference of square values between each $m c r$ - or $d s r$-containing contig and its host MAG, and also genomes of their related methanogens and archaea. Completeness and contamination of each MAG was assessed with CheckM $^{39}$ version 1.0.7 using lineage-specific (149-228) and archaea (149) marker genes. ORFs of these MAGs were predicted with Prodigal ${ }^{34}$ version 2.6.3. The predicted ORFs were searched against the NCBI nr protein database (March 2017) and eggNOG ${ }^{42}$ database with the BLASTP algorithm (coverage $>75 \%$ and $e<1 \times 10^{-20}$ ) to check their protein identities to the most closely related sequences using DIAMOND version 0.8.28.90. Specifically, the NCBI nr protein database and eggNOG database were downloaded and local protein databases were constructed. Then, the MAG protein sequences were searched against the established databases as query. The highest-scored sequences from the two databases were cross-checked and sequences related to MCR-based alkane metabolism were also manually checked by BLASTP search on the NCBI website (https://blast.ncbi.nlm.nih.gov/Blast.cgi). The HydDB was first downloaded and a local database was established. Then, all predicted protein sequences from the MAGs were queried against the local HydDB with parameters coverage $>75 \%$ and $e<1 \times 10^{-20}$. Next, the result sequences were searched again in the NCBI nr database for further confirmations. For metabolic pathway analyses, we used the web portal GhostKOALA on the $\mathrm{KEGG}^{43}$ website. The AAI values were generated by compareM version 0.0 .23 with parameters aai_wf and --proteins (https://github.com/dparks1134/CompareM). The taxonomic assignments of MAGs were based on criteria provided in references ${ }^{44-47}$.

Phylogenetic analyses based on conserved proteins, 16S rRNA genes and McrABG/DsrA/AprA subunits. For phylogenomic analysis, 233 representative archaea reference genomes from the superphyla Euryarchaeota, TACK, Asgard and DPANN were downloaded from the NCBI prokaryote genome database (https:// www.ncbi.nlm.nih.gov/assembly/; the genome list can be found in https://figshare. com/, https://doi.org/10.6084/m9.figshare.7149623). These reference genomes and the MAGs from this study were used to construct a phylogenomic tree based on a concatenated alignment of a set of 38 marker genes as suggested by Hug et al. ${ }^{48}$ (Supplementary Table 5). Nevertheless, the removal of the protein sequence translation elongation factor GTPase affects the placement of the Hadesarchaeota in the phylogenomic tree; thus, the sequence of the translation elongation factor GTPase was not considered in further phylogenomic analysis. Specifically, each of the 37 marker protein sequences from the reference genomes and the MAGs was aligned using the MAFFT $^{49}$ algorithm version 7.313 with parameters --ep 0 --genafpair --maxiterate 1,000 and filtered with trim $\mathrm{Al}^{50}$ version 1.4.rev2 with parameter -automated 1 . Then, all 37 marker genes were concatenated into a single alignment and phylogenetic trees were built using both IQ-Tree ${ }^{51}$ version 1.6.6 with the model $\mathrm{LG}+\mathrm{C} 60+\mathrm{F}+\mathrm{G}$ and $\mathrm{RAxML}{ }^{52}$ version 8.0 with the model PROTGAMMAAUTO with a bootstrap value of 1,000 . For the phylogenetic analysis of functional marker proteins (McrA, McrB, McrG, DsrA and AprA) and the 16S rRNA gene, the respective protein and nucleotide sequences were retrieved from the MAGs, and additional reference sequences were obtained from the NCBI (https://www.ncbi.nlm.nih.gov/protein/). Alignment and filtering was carried out with the same programs described above. For $16 \mathrm{~S}$ rRNA gene sequences, phylogenetic trees were built using both IQ-Tre ${ }^{51}$ version 1.6.6 with the model
$\mathrm{HKY}+\mathrm{F}+\mathrm{G}$ and $\mathrm{RAxML}{ }^{52}$ version 8.0 with the model GTRGAMMA with a bootstrap value of 1,000 .

Reporting Summary. Further information on research design is available in the Nature Research Reporting Summary linked to this article.

\section{Code availability}

All scripts and analyses necessary to perform metagenome processing can be accessed from GitHub (https://github.com/) or the websites provided in the original research articles. The specific links to the custom software are listed below: DIAMOND version 0.8.28.90: http://ab.inf.uni-tuebingen.de/software/ diamond/, Sickle version 1.33: https://github.com/najoshi/sickle, MEGAHIT version 1.0.6-hotfix1: https://hku-bal.github.io/megabox/, Bowtie version 2.2.8: http://bowtie-bio.sourceforge.net/bowtie2/index.shtml, Prodigal version 2.6.3: http://compbio.ornl.gov/prodigal/, MaxBin version 2.2.4: http://sourceforge.net/ projects/maxbin/, MetaBAT version 2.12.1: https://bitbucket.org/berkeleylab/ metabat, CheckM version 1.0.7: http://ecogenomics.github.io/CheckM, compareM version 0.0.23: https://github.com/dparks1134/CompareM, MAFFT version 7.313: https://mafft.cbrc.jp/alignment/software/, trimAl version 1.4.rev2: http://trimal. cgenomics.org, IQ-Tree version 1.6.6: http://www.cibiv.at/software/iqtree, and RAxML version 8.0: https://github.com/stamatak/standard-RAxML.

\section{Data availability}

The data sets generated and/or analysed during the current study are available in the NCBI repository at https://www.ncbi.nlm.nih.gov/. The MAGs from the current study have been deposited in the NCBI GenBank under the project ID PRJNA475886.

Received: 19 June 2018; Accepted: 7 January 2019;

Published online: 04 March 2019

\section{References}

1. Ueno, Y., Yamada, K., Yoshida, N., Maruyama, S. \& Isozaki, Y. Evidence from fluid inclusions for microbial methanogenesis in the early Archaean era. Nature 440, 516-519 (2006).

2. Conrad, R. The global methane cycle: recent advances in understanding the microbial processes involved. Environ. Microbiol. Rep. 1, 285-292 (2009).

3. Reeburgh, W. Oceanic methane biogeochemistry. Chem. Rev. 107, 486-513 (2007).

4. Knittel, K. \& Boetius, A. Anaerobic oxidation of methane: progress with an unknown process. Annu. Rev. Microbiol. 63, 311-334 (2009).

5. Krüger, M. et al. A conspicuous nickel protein in microbial mats that oxidize methane anaerobically. Nature 426, 878-881 (2003).

6. Hallam, S. J. et al. Reverse methanogenesis: testing the hypothesis with environmental genomics. Science 305, 1457-1462 (2004).

7. Scheller, S., Goenrich, M., Boecher, R., Thauer, R. K. \& Jaun, B. The key nickel enzyme of methanogenesis catalyses the anaerobic oxidation of methane. Nature 465, 606-608 (2010).

8. Gunsalus, R. \& Wolfe, R. Methyl coenzyme M reductase from Methanobacterium thermoautotrophicum. Resolution and properties of the components. J. Biol. Chem. 255, 1891-1895 (1980).

9. Ermler, U., Grabarse, W., Shima, S., Goubeaud, M. \& Thauer, R. K. Crystal structure of methyl-coenzyme $M$ reductase: the key enzyme of biological methane formation. Science 278, 1457-1462 (1997).

10. Thauer, R. K. Anaerobic oxidation of methane with sulfate: on the reversibility of the reactions that are catalyzed by enzymes also involved in methanogenesis from $\mathrm{CO}_{2}$. Curr. Opin. Microbiol. 14, 292-299 (2011).

11. Lever, M. A. \& Teske, A. P. Diversity of methane-cycling archaea in hydrothermal sediment investigated by general and group-specific PCR primers. Appl. Environ. Microbiol. 81, 1426-1441 (2015).

12. Evans, P. N. et al. Methane metabolism in the archaeal phylum Bathyarchaeota revealed by genome-centric metagenomics. Science 350, 434-438 (2015)

13. Vanwonterghem, I. et al. Methylotrophic methanogenesis discovered in the archaeal phylum Verstraetearchaeota. Nat. Microbiol. 1, 16170 (2016).

14. Laso-Pérez, R. et al. Thermophilic archaea activate butane via alkyl-coenzyme M formation. Nature 539, 396-401 (2016)

15. Boetius, A. et al. A marine microbial consortium apparently mediating anaerobic oxidation of methane. Nature 407, 623-626 (2000).

16. McGlynn, S. E., Chadwick, G. L., Kempes, C. P. \& Orphan, V. J. Single cell activity reveals direct electron transfer in methanotrophic consortia. Nature 526, 531-535 (2015).

17. Wegener, G., Krukenberg, V., Riedel, D., Tegetmeyer, H. E. \& Boetius, A. Intercellular wiring enables electron transfer between methanotrophic archaea and bacteria. Nature 526, 587-590 (2015).

18. Haroon, M. F. et al. Anaerobic oxidation of methane coupled to nitrate reduction in a novel archaeal lineage. Nature 500, 567-570 (2013). 
19. Ettwig, K. F. et al. Archaea catalyze iron-dependent anaerobic oxidation of methane. Proc. Natl Acad. Sci. USA 113, 12792-12796 (2016).

20. Baker, B. J. et al. Genomic inference of the metabolism of cosmopolitan subsurface Archaea, Hadesarchaea. Nat. Microbiol. 1, 16002 (2016).

21. Elkins, J. G. et al. A korarchaeal genome reveals insights into the evolution of the Archaea. Proc. Natl Acad. Sci. USA 105, 8102-8107 (2008).

22. Adam, P. S., Borrel, G., Brochier-Armanet, C. \& Gribaldo, S. The growing tree of Archaea: new perspectives on their diversity, evolution and ecology. ISME J. 11, 2407-2425 (2017).

23. Parks, D. H. et al. A standardized bacterial taxonomy based on genome phylogeny substantially revises the tree of life. Nat. Biotechnol. 36, 996-1004 (2018)

24. Raymann, K., Brochier-Armanet, C. \& Gribaldo, S. The two-domain tree of life is linked to a new root for the Archaea. Proc. Natl Acad. Sci. USA 112 6670-6675 (2015).

25. Petitjean, C., Deschamps, P., López-García, P., Moreira, D. \& BrochierArmanet, C. Extending the conserved phylogenetic core of archaea disentangles the evolution of the third domain of life. Mol. Biol. Evol. 32, 1242-1254 (2015)

26. Williams, T. A. et al. Integrative modeling of gene and genome evolution roots the archaeal tree of life. Proc. Natl Acad. Sci. USA 114, E4602-E4611 (2017).

27. Stahl, D. A., Fishbain, S., Klein, M., Baker, B. J. \& Wagner, M. Origins and diversification of sulfate-respiring microorganisms. Antonie Van Leeuwenhoek 81, 189-195 (2002).

28. Müller, A. L., Kjeldsen, K. U., Rattei, T., Pester, M. \& Loy, A. Phylogenetic and environmental diversity of DsrAB-type dissimilatory (bi) sulfite reductases. ISME J. 9, 1152-1165 (2015).

29. Timmers, P. H. et al. Reverse methanogenesis and respiration in methanotrophic archaea. Archaea 2017, 1654237 (2017).

30. Stojanowic, A., Mander, G. J., Duin, E. C. \& Hedderich, R. Physiological role of the F420-non-reducing hydrogenase (Mvh) from Methanothermobacter marburgensis. Arch. Microbiol. 180, 194-203 (2003).

31. Buchfink, B., Xie, C. \& Huson, D. H. Fast and sensitive protein alignment using DIAMOND. Nat. Methods 12, 59-60 (2014).

32. Li, D., Liu, C.-M., Luo, R., Sadakane, K. \& Lam, T.-W. MEGAHIT: an ultra-fast single-node solution for large and complex metagenomics assembly via succinct de Bruijn graph. Bioinformatics 31, 1674-1676 (2015).

33. Langmead, B. \& Salzberg, S. L. Fast gapped-read alignment with Bowtie 2. Nat. Methods 9, 357-359 (2012).

34. Hyatt, D. et al. Prodigal: prokaryotic gene recognition and translation initiation site identification. BMC Bioinformatics 11, 119 (2010).

35. Kanehisa, M., Furumichi, M., Tanabe, M., Sato, Y. \& Morishima, K. KEGG: new perspectives on genomes, pathways, diseases and drugs. Nucleic Acids Res. 45, D353-D361 (2016).

36. Dick, G. J. et al. Community-wide analysis of microbial genome sequence signatures. Genome Biol. 10, R85 (2009).

37. Wu, Y.-W., Simmons, B. A. \& Singer, S. W. MaxBin 2.0: an automated binning algorithm to recover genomes from multiple metagenomic datasets. Bioinformatics 32, 605-607 (2015).

38. Kang, D. D., Froula, J., Egan, R. \& Wang, Z. MetaBAT, an efficient tool for accurately reconstructing single genomes from complex microbial communities. PeerJ 3, el165 (2015).

39. Parks, D. H., Imelfort, M., Skennerton, C. T., Hugenholtz, P. \& Tyson, G. W. CheckM: assessing the quality of microbial genomes recovered from isolates, single cells, and metagenomes. Genome Res. 25, 1043-1055 (2015).

40. Karst, S. M., Kirkegaard, R. H. \& Albertsen, M. mmgenome: a toolbox for reproducible genome extraction from metagenomes. Preprint at https://doi. org/10.1101/059121 (2016).

41. Bowers, R. M. et al. Minimum information about a single amplified genome (MISAG) and a metagenome-assembled genome (MIMAG) of bacteria and archaea. Nat. Biotechnol. 35, 725-731 (2017).

42. Huerta-Cepas, J. et al. eggNOG 4.5: a hierarchical orthology framework with improved functional annotations for eukaryotic, prokaryotic and viral sequences. Nucleic Acids Res. 44, D286-D293 (2016).
43. Kanehisa, M., Sato, Y. \& Morishima, K. BlastKOALA and GhostKOALA: KEGG tools for functional characterization of genome and metagenome sequences. J. Mol. Biol. 428, 726-731 (2016).

44. Yarza, P. et al. Uniting the classification of cultured and uncultured bacteria and archaea using 16S rRNA gene sequences. Nat. Rev. Microbiol. 12, 635-645 (2014)

45. Hugenholtz, P., Skarshewski, A. \& Parks, D. H. Genome-based microbial taxonomy coming of age. Cold Spring Harb. Perspect. Biol. 8 , a018085 (2016)

46. Konstantinidis, K. T., Rosselló-Móra, R. \& Amann, R. Uncultivated microbes in need of their own taxonomy. ISME J. 11, 2399-2406 (2017).

47. Chuvochina, M. et al. The importance of designating type material for uncultured taxa. Syst. Appl. Microbiol. https://doi.org/10.1016/j. syapm.2018.07.003 (2018)

48. Hug, L. A. et al. A new view of the tree of life. Nat. Microbiol. 1, 16048 (2016)

49. Katoh, K. \& Standley, D. M. MAFFT multiple sequence alignment software version 7: improvements in performance and usability. Mol. Biol. Evol. 30 772-780 (2013)

50. Capella-Gutiérrez, S., Silla-Martínez, J. M. \& Gabaldón, T. trimAl: a tool for automated alignment trimming in large-scale phylogenetic analyses. Bioinformatics 25, 1972-1973 (2009).

51. Stamatakis, A. RAxML version 8: a tool for phylogenetic analysis and post-analysis of large phylogenies. Bioinformatics 30, 1312-1313 (2014).

52. Nguyen, L.-T., Schmidt, H. A., von Haeseler, A. \& Minh, B. Q. IQ-TREE: a fast and effective stochastic algorithm for estimating maximum-likelihood phylogenies. Mol. Biol. Evol. 32, 268-274 (2014).

53. Jungbluth, S. P., Amend, J. P. \& Rappé, M. S. Metagenome sequencing and 98 microbial genomes from Juan de Fuca Ridge flank subsurface fluids. Sci. Data 4, 170037 (2017).

\section{Acknowledgements}

We thank R. K. Thauer for his advice on the discussed metabolic pathways, and V. Krukenberg and J. Wang for valuable discussion and suggestions for the manuscript We are grateful to the researchers who published their sequence data on the NCBI (https://www.ncbi.nlm.nih.gov/), and to the US Department of Energy Joint Genome Institute (http://www.jgi.doe.gov/) for providing protein sequence files in collaboration with the user community. We thank the following sources for funding: the Natural Science Foundation of China (grant numbers 91751205, 41525011 and 91428308), the National Key R\&D project of China (grant number 2018YFC0309800) and China Postdoctoral Science Foundation Grant (grant number 2018T110390). This study is also a contribution to the Deep Carbon Observatory.

\section{Author contributions}

Y.W. and F.W. designed the research, performed the analyses, developed the metabolic models and wrote the paper. G.W. developed the metabolic models and wrote the paper J.H. provided useful discussion and helped with the double-blind assessments of the MAGs. F.W. and X.X. provided guidance and useful suggestion.

\section{Competing interests}

The authors declare no competing interests.

\section{Additional information}

Supplementary information is available for this paper at https://doi.org/10.1038/ s41564-019-0364-2.

Reprints and permissions information is available at www.nature.com/reprints. Correspondence and requests for materials should be addressed to F.W. or X.X. Publisher's note: Springer Nature remains neutral with regard to jurisdictional claims in published maps and institutional affiliations.

(c) The Author(s), under exclusive licence to Springer Nature Limited 2019 


\title{
Reporting Summary
}

Nature Research wishes to improve the reproducibility of the work that we publish. This form provides structure for consistency and transparency in reporting. For further information on Nature Research policies, see Authors \& Referees and the Editorial Policy Checklist.

\section{Statistical parameters}

When statistical analyses are reported, confirm that the following items are present in the relevant location (e.g. figure legend, table legend, main text, or Methods section).
n/a Confirmed
$\square \bigotimes$ The exact sample size $(n)$ for each experimental group/condition, given as a discrete number and unit of measurement
Х $\square$ An indication of whether measurements were taken from distinct samples or whether the same sample was measured repeatedly
\ $\square$ The statistical test(s) used AND whether they are one- or two-sided
$\square$ Only common tests should be described solely by name; describe more complex techniques in the Methods section.
Х $\square$ A description of all covariates tested
Х $\square$ A description of any assumptions or corrections, such as tests of normality and adjustment for multiple comparisons
$\triangle \square$ A full description of the statistics including central tendency (e.g. means) or other basic estimates (e.g. regression coefficient) AND $\searrow \square$ variation (e.g. standard deviation) or associated estimates of uncertainty (e.g. confidence intervals)
For null hypothesis testing, the test statistic (e.g. $F, t, r$ ) with confidence intervals, effect sizes, degrees of freedom and $P$ value noted
Х $\square$ Give $P$ values as exact values whenever suitable.
Х $\square$ For Bayesian analysis, information on the choice of priors and Markov chain Monte Carlo settings
\ $\square$ For hierarchical and complex designs, identification of the appropriate level for tests and full reporting of outcomes
Х $\square$ Estimates of effect sizes (e.g. Cohen's $d$, Pearson's $r$ ), indicating how they were calculated
Clearly defined error bars
State explicitly what error bars represent (e.g. SD, SE, CI)

Our web collection on statistics for biologists may be useful.

\section{Software and code}

Policy information about availability of computer code
Data collection
No software was used to collect data.

Data analysis

\begin{abstract}
All scripts and softwares to perform metagenome assembly, annotation and binning can be accessed from
github (https://github.com/) or are listed in methods with versions and can be downloaded from the websites provided in the publications in the references part in the manuscript. The specific links to the custom software are listed below: DIAMOND version 0.8.28.90: http://ab.inf.uni-tuebingen.de/software/diamond/, Sickle version 1.33: https://github.com/najoshi/sickle, MEGAHIT version 1.0.6-hotfix1: https://hku-bal.github.io/megabox/, Bowtie version 2.2.8: http://bowtie-bio.sourceforge.net/bowtie2/index.shtml, Prodigal version 2.6.3: http://compbio.ornl.gov/prodigal/, MaxBin version 2.2.4: http://sourceforge.net/projects/maxbin/, MetaBAT version 2.12.1: https://bitbucket.org/berkeleylab/metabat, CheckM version 1.0.7: http://ecogenomics.github.io/CheckM, compareM version 0.0.23: https://github.com/dparks1134/CompareM, MAFFT version 7.313: https://mafft.cbrc.jp/alignment/software/, trimAl version 1.4.rev2: http://trimal.cgenomics.org, IQ-Tree version 1.6.6: http://www.cibiv.at/software/iqtree, RAxML version 8.0: https:// github.com/stamatak/standard-RAxML.
\end{abstract}


Policy information about availability of data

All manuscripts must include a data availability statement. This statement should provide the following information, where applicable:

- Accession codes, unique identifiers, or web links for publicly available datasets

- A list of figures that have associated raw data

- A description of any restrictions on data availability

The datasets generated and/or analysed during the current study are available in the NCBI repository at https://www.ncbi.nlm.nih.gov/. The accession numbers are listed in Supplementary table 2. The genomic bins from the current study have been deposited in NCBI GenBank under the project ID PRJNA475886.

\section{Field-specific reporting}

Please select the best fit for your research. If you are not sure, read the appropriate sections before making your selection.

Life sciences

Behavioural \& social sciences

Ecological, evolutionary \& environmental sciences

For a reference copy of the document with all sections, see nature.com/authors/policies/ReportingSummary-flat.pdf

\section{Ecological, evolutionary \& environmental sciences study design}

All studies must disclose on these points even when the disclosure is negative.

Study description

We show that methyl-CoM reductase (MCR) based alkane metabolism is present in a wide array of phyla of the domain Archaea through a global survey of MCR-encoding genes in public metagenomic data from various environments. Hydrothermally heated environments were found to contain mostly novel types of mcr genes, and eleven high-quality mcr-containing genomic bins were obtained belonging to a variety of archaeal phyla, including the Archaeoglobi in the Euryarchaeota, the Hadesarchaeota, and different TACK superphylum archaea, including the novel Nezhaarchaeota, Korarchaeota and recently described Verstraetearchaeota. The Archaeoglobi here and a novel strain of Korarchaeota combine the genes for (reverse) methanogenesis and reductive sulfur metabolism, suggesting a coupling of methane oxidation with sulfate reduction in individual organisms.

Research sample

The data used in the current study are existing shotgun metagenome sequence data from natural environments in public databases, including marine sediment, hot springs, freshwater niches. All metagenomes raw data analyzed here are available in the NCBI repository at https://www.ncbi.nlm.nih.gov/. The accession numbers of the detests are listed in Supplementary table 2. Specifically, the geothermal environment metagenomic data sets were assembled and binned. Previously unidentified alkane metabolism archaea were found within these data sets.

Sampling strategy

In the current study, sampling strategy is not applicable because we mainly focused on discovery of potential alkane metabolism archaea in natural environments and we screened all JGI metagenomic protein database while only took the samples with McrA protein sequences. We then selected the mcrA gene containing metagenomic datasets from public available NCBI SRA database for further analyses.

Data collection

The first author Yinzhao Wang collected all datasets from the NCBI SRA repository and did the analyses.

Timing and spatial scale

The data collection started/end at Jan. 2017

Data exclusions

No data were excluded from the analyses.

Reproducibility

No experiment was carried out in the current study. The reproducibility can be conducted by following the methods for shotgun metagenome sequence data as mentioned in the main text.

Randomization No randomization was made because no statistical analyses were performed here. We mainly focused on discovery of potential alkane metabolism archaea in natural environments.

Blinding

Blinding was not relevant to this study, given that it primarily involved the analyses of metagenomic datasets.

Did the study involve field work? $\square$ Yes $\quad$ No

\section{Reporting for specific materials, systems and methods}


Materials \& experimental systems

$\mathrm{n} / \mathrm{a}$ Involved in the study

\ $\square$ Unique biological materials

Х $\square$ Antibodies

\ $\square$ Eukaryotic cell lines

Х $\square$ Palaeontology

$\triangle \square$ Animals and other organisms

$\bigotimes \mid \square$ Human research participants
Methods

$\mathrm{n} / \mathrm{a}$ Involved in the study

$\triangle(\square$ ChIP-seq

Х $\square$ Flow cytometry

$\searrow \mid \square$ MRI-based neuroimaging 